Article

\title{
Study on the Existence of Solutions for a Class of Nonlinear Neutral Hadamard-Type Fractional Integro-Differential Equation with Infinite Delay
}

\author{
Kaihong Zhao * and Yue Ma
}

Citation: Zhao, K.; Ma, Y. Study on The Existence of Solutions for a Class of Nonlinear Neutral

Hadamard-Type Fractional Integro-Differential Equation with Infinite Delay. Fractal Fract. 2021, 5, 52. https://doi.org/10.3390/ fractalfract5020052

Academic Editor: Ivanka Stamova

Received: 22 May 2021

Accepted: 1 June 2021

Published: 4 June 2021

Publisher's Note: MDPI stays neutral with regard to jurisdictional claims in published maps and institutional affiliations.

Copyright: (c) 2021 by the authors. Licensee MDPI, Basel, Switzerland. This article is an open access article distributed under the terms and conditions of the Creative Commons Attribution (CC BY) license (https:/ / creativecommons.org/licenses/by/ $4.0 /)$.
Department of Mathematics, Kunming University of Science and Technology, Kunming 650500, China; mayue_twinkle@163.com

* Correspondence: zhaokaihongs@126.com

Abstract: The existence of solutions for a class of nonlinear neutral Hadamard-type fractional integrodifferential equations with infinite delay is researched in this paper. By constructing an appropriate normed space and utilizing the Banach contraction principle, Krasnoselskii's fixed point theorem, we obtain some sufficient conditions for the existence of solutions. Finally, we provide an example to illustrate the validity of our main results.

Keywords: Hadamard fractional derivative; neutral fractional equations; existence; infinite delay; fixed point theory

MSC: 34A08; 34K37; 34K40

\section{Introduction}

The main purpose of this paper is to study the existence of solutions for the following Hadamard-type neutral fractional integro-differential equation with infinite delay written by

$$
\left\{\begin{array}{l}
{ }^{H} D_{1^{+}}^{\alpha}\left[u(t)-\sum_{i=1}^{m} H J_{1^{+}}^{\beta_{i}} e_{i}\left(t, u_{t}\right)\right]=a(t) f\left(t, u_{t}, \int_{1}^{t} g\left(t, s, u_{s}\right) d s\right), t \in J=[1, T], \\
u(t)=\phi(t), \quad t \in(-\infty, 1],
\end{array}\right.
$$

where $T>1,0<\alpha \leq 1$ and $\beta_{i}>0(i=1,2, \ldots, m)$ are some given real constants. ${ }^{H} D_{1+}^{\alpha}$ denotes the Hadamard-type fractional derivative of order $\alpha,{ }^{H} J_{1+}^{\beta_{i}}$ stands the Hadamard fractional integral of order $\beta_{i} . e_{i}: J \times \mathcal{B}_{h} \rightarrow \mathbb{R}, f: J \times \mathcal{B}_{h} \times \mathbb{R} \rightarrow \mathbb{R}$ and $g: J \times J \times \mathcal{B}_{h} \rightarrow \mathbb{R}$ are given continuous functions satisfying some assumptions that will be introduced later. The function $a(t) \in C[J, \mathbb{R}]$ and it is not identically zero on any subinterval of $[1, T]$. We also assume that $u_{t}:(-\infty, 0] \rightarrow \mathcal{B}_{h}$ with $u_{t}(\theta)=u(t+\theta)(\theta \leq 0)$ belongs to an abstract phase space $\mathcal{B}_{h}$ which is defined in Preliminaries. $\phi(t):(-\infty, 1] \rightarrow \mathbb{R}$ is a given continuous initial function with $\phi(1)=0$.

Fractional calculus, especially fractional differential equation is an advantageous mathematical model used to describe properties of various processes and applications in lots of fields. Therefore, the fractional differential equations are widely concerned and studied by many people. There have been many papers dealing with fractional differential equations with different conditions such as impulses [1-6], time delays [1,3,4,7,8], implicit conditions [9], algebraic conditions [10], stochastic conditions [4], neutral conditions $[1,2,6,10,11]$, boundary value conditions [5,7-9,12], and so on. In 1892, Hadamard [13] proposed a new kind of fractional calculus. The definition of this fractional calculus contains the logarithmic function of any index in the integral kernel. This kind of fractional calculus is later called Hadamard fractional calculus. Hadamard fractional calculus has been widely concerned and studied 
by many scholars since it was put forward. In the monographs [14,15], the authors introduced the basic knowledge and results of Hadamard's fractional calculus and differential equation in detail. Some researchers have studied and explored the dynamic properties of Hadamard fractional calculus and differential equations. For example, the Hadamard fractional integration operators and the semigroup property were discussed in [16]. The Mellin's transformation of Hadamard fractional calculus was investigated in [17]. Some papers on Hadamard fractional differential boundary value problems [5,18-23], integrodifferential equations [5,18,24], neutral equations [24] and coupled systems [5,25] have been published. Compared with the research results of Riemann-Liouville and Caputo fractional differential equations, the study on Hadamard fractional differential equation are relatively rare. Therefore, it is valuable and challenging to study the dynamic properties of Hadamard fractional differential equations.

In addition, the research on system (1) is also inspired by the literature [24]. The author studied the existence and uniqueness results of solutions for the following system

$$
\left\{\begin{array}{l}
{ }^{H} D^{\alpha}\left[u(t)-\sum_{i=1}^{m} I^{\beta_{i}} h_{i}\left(t, u_{t}\right)\right]=f\left(t, u_{t}\right), \quad t \in J=[1, T], \\
u(t)=\varphi(t), t \in[1-r, 1], r>0
\end{array}\right.
$$

where $T>1,0<\alpha \leq 1$ and $\beta_{i}>0(i=1,2, \ldots, m)$ are some given real constants. ${ }^{H} D^{\alpha}$ denotes the left-side Hadamard fractional derivative of order $\alpha, I^{\beta_{i}}$ stands the RiemannLiouville fractional integral of order $\beta_{i}>0 . f, h_{i}: J \times C([-r, 0], \mathbb{R}) \rightarrow \mathbb{R}$ are given continuous functions, $\varphi \in C([1-r, 1], \mathbb{R})$ with $\varphi(1)=0$. For any $u$, it is a function defined on $[1-r, T]$ and for any $t \in J, u_{t}(\theta)=u(t+\theta) \in C([-r, 0], \mathbb{R}), \theta \in[-r, 0]$. The author established the uniqueness of solutions by the Banach contraction principle and derived the existence of solutions by the Leray-Schauder alternative.

The rest of the paper is organized as follows. In Section 2, we recall some useful definitions, notations and lemmas. The main results are proved in Section 3. In Section 4, an example is given to demonstrate the application of our main results. Finally, we conclude the importance of studying problem (1) and our obtained results in Section 5.

\section{Preliminaries}

In this section, we introduce some necessary definitions, notations, lemmas and preliminary facts, which are required in the sequel.

First of all, we present the abstract phase $\mathcal{B}_{h}$ similar to [26]. Here we assume that $h:(-\infty, 0] \rightarrow(0,+\infty)$ is a continuous function with $l=\int_{-\infty}^{0} h(t) d t<+\infty$. For any $c>0$, we define $\mathcal{B}=C([-c, 0], \mathbb{R})$ equipped with the norm $\|\psi\|_{[-c, 0]}=\sup _{s \in[-c, 0]}|\psi(s)|, \forall \psi \in$ $\mathcal{B}$. Define

$$
\left.\mathcal{B}_{h}=\{\psi \mid \psi \in C(-\infty, 0], \mathbb{R}) \text { such that for any } b>0,\left.\psi\right|_{[-b, 0]} \in \mathcal{B} \text { and } \int_{-\infty}^{0} h(s)\|\psi\|_{[s, 0]} d s<+\infty\right\} \text {. }
$$

Clearly, $\left(\mathcal{B}_{h},\|\cdot\|_{\mathcal{B}_{h}}\right)$ is a Banach space equipped with the norm

$$
\|\psi\|_{\mathcal{B}_{h}}=\int_{-\infty}^{0} h(s)\|\psi\|_{[s, 0]} d s, \forall \psi \in \mathcal{B}_{h} .
$$

Define

$$
\left.\mathcal{B}_{h}^{\prime}=\{w \mid w \in C(-\infty, T], \mathbb{R}) \text { such that } w(1)=\phi(1)=0\right\}
$$

equipped with $\|w\|_{\mathcal{B}_{h}^{\prime}}=\max \left\{\|\phi\|_{\infty},\|w\|_{T}\right\}$, where $\|\phi\|_{\infty}=\max \left\{\|\phi\|_{\mathcal{B}_{h}}, \sup _{t \in[0,1]}|\phi(t)|\right\}$, $\|w\|_{T}=\sup _{s \in[1, T]}|w(s)|$. Let $\mathbf{X}=\mathcal{B}_{h^{\prime}}^{\prime}$, for any $u \in \mathbf{X}$, define $\|u\|=\|u\|_{\mathcal{B}_{h}^{\prime}}$, it is easy to verify that $\mathbf{X}$ is a Banach space. 
Definition 1 ([14]). The left-sided Hadamard fractional integral of order $\alpha>0$ for a function $u:[1, \infty) \rightarrow \mathbb{R}$ is defined by

$$
{ }^{H} J_{1^{+}}^{\alpha} u(t)=\frac{1}{\Gamma(\alpha)} \int_{1}^{t}\left(\ln \frac{t}{s}\right)^{\alpha-1} u(s) \frac{d s}{s},
$$

provided the integral exists.

Definition 2 ([14]). Let $u \in C^{n}([1, \infty))$. Then the Hadamard type fractional derivative of order $\alpha>0$ for a function $u:[1, \infty) \rightarrow \mathbb{R}$ is defined by

$$
{ }^{H} D_{1^{+}}^{\alpha} u(t)=\frac{1}{\Gamma(n-\alpha)}\left(t \frac{d}{d t}\right)^{n} \int_{1}^{t}\left(\ln \frac{t}{s}\right)^{n-\alpha-1} u(s) \frac{d s}{s}, n-1<\alpha \leq n, n=[\alpha]+1,
$$

where $[\alpha]$ denotes the integer part of the real number $\alpha>0$, and $\Gamma(\cdot)$ is the Gamma function.

Lemma 1 ([14]). Assume that $u \in C^{n}(1, T) \cap L^{1}(1, T)$ with a Hadamard type fractional derivative of order $\alpha>0$. Then

$$
{ }^{H} J_{1^{+}}^{\alpha}{ }^{H} D_{1^{+}}^{\alpha} u(t)=u(t)+c_{1}(\ln t)^{\alpha-1}+c_{2}(\ln t)^{\alpha-2}+\ldots+c_{n}(\ln t)^{\alpha-n},
$$

where $c_{i} \in \mathbb{R}, i=1,2, \ldots, n-1, n$ and $n=[\alpha]+1$.

Lemma 2 ([14]). If $\alpha>0, \beta>0$, then the following properties hold:

$$
\begin{aligned}
& { }^{H} D_{1^{+}}^{\alpha}(\ln t)^{\beta-1}=\frac{\Gamma(\beta)}{\Gamma(\beta-\alpha)}(\ln t)^{\beta-\alpha-1}, \\
& { }^{H} J_{1^{+}}^{\alpha}(\ln t)^{\beta-1}=\frac{\Gamma(\beta)}{\Gamma(\beta+\alpha)}(\ln t)^{\beta+\alpha-1}, \\
& { }^{H} J_{1^{+}}^{\alpha}{ }^{H} D_{1^{+}}^{\alpha} u(t)=u(t),
\end{aligned}
$$

in particular, ${ }^{H} D_{1^{+}}^{\alpha}(\ln t)^{\alpha-j}=0, j=1,2, \ldots,[\alpha]+1$.

Lemma 3 (Banach contraction principle [27]). If $E$ is a real Banach space and $F: E \rightarrow E$ is a contraction mapping, then $F$ has a unique fixed point in $E$.

Lemma 4 (Krasnoselskii's fixed point theorem [28]). Let $X_{1}$ be a nonempty closed convex subset of a Banach space $(\mathbf{X},\|\cdot\|)$. Let $P, Q$ be two operators such that

(i) $P x+Q y \in X_{1}$ for any $x, y \in X_{1}$;

(ii) $P$ is a contraction mapping;

(iii) $Q$ is continuous and compact.

Then there exists $a z \in X_{1}$ such that $z=P z+Q z$.

Lemma 5. Let $\xi(t), \eta(t) \in C(J, \mathbb{R})$ and $\phi(t) \in((-\infty, 1], \mathbb{R})$ with $\phi(1)=0$ be some given functions, and $0<\alpha \leq 1, \beta>0$ and $T>1$ be some constants. Then a function $u(t) \in \mathbf{X}$ is a solution of the following linear system

$$
\left\{\begin{array}{l}
{ }^{H} D_{1^{+}}^{\alpha}\left[u(t)-{ }^{H} J_{1^{+}}^{\beta} \eta(t)\right]=\xi(t), t \in J=[1, T] \\
u(t)=\phi(t), t \in(-\infty, 1]
\end{array}\right.
$$


if and only if $u(t) \in \mathbf{X}$ is a solution of the integral equation as follows:

$$
u(t)=\left\{\begin{array}{l}
\frac{1}{\Gamma(\alpha)} \int_{1}^{t}\left(\ln \frac{t}{s}\right)^{\alpha-1} \xi(s) \frac{d s}{s}+{ }^{H} J_{1^{+}}^{\beta} \eta(t), \quad t \in J=[1, T], \\
\phi(t), \quad t \in(-\infty, 1] .
\end{array}\right.
$$

Proof. Assume that $u(t) \in \mathbf{X}$ is a solution of (2). When $t \in J=[1, T]$, according to the first equation of (2) and Lemma 1, we have

$$
u(t)-{ }^{H} J_{1^{+}}^{\beta} \eta(t)=\frac{1}{\Gamma(\alpha)} \int_{1}^{t}\left(\ln \frac{t}{s}\right)^{\alpha-1} \xi(s) \frac{d s}{s}+c_{1}(\ln t)^{\alpha-1} .
$$

In the light of the existence of $u(1)=0$, we have $c_{1}=0$. When $t \in(-\infty, 1]$, then $u(t)=\phi(t)$. Thus, we derive

$$
u(t)=\left\{\begin{array}{l}
\frac{1}{\Gamma(\alpha)} \int_{1}^{t}\left(\ln \frac{t}{s}\right)^{\alpha-1} \xi(s) \frac{d s}{s}+{ }^{H} J_{1^{+}}^{\beta} \eta(t), \quad t \in J=[1, T] \\
\phi(t), t \in(-\infty, 1],
\end{array}\right.
$$

which indicates that $u(t) \in \mathbf{X}$ is also a solution of (3). Conversely, if $u(t) \in \mathbf{X}$ is a solution of (3), noting that the above process is completely reversible, then we know that $u(t) \in \mathbf{X}$ is also a solution of (2). The proof is completed.

\section{Main Results}

For any $t \in(-\infty, T], e_{i}: J \times \mathcal{B}_{h} \rightarrow \mathbb{R}, f: J \times \mathcal{B}_{h} \times \mathbb{R} \rightarrow \mathbb{R}, g: J^{2} \times \mathcal{B}_{h} \rightarrow \mathbb{R}, u(t) \in \mathbf{X}$, it follows from Lemma 5 that an operator $F: \mathbf{X} \rightarrow \mathbf{X}$ defined by

$$
(F u)(t)=\left\{\begin{array}{l}
\frac{1}{\Gamma(\alpha)} \int_{1}^{t}\left(\ln \frac{t}{s}\right)^{\alpha-1} a(s) f\left(s, u_{s}, \int_{1}^{s} g\left(s, \tau, u_{\tau}\right) d \tau\right) \frac{d s}{s}+\sum_{i=1}^{m}{ }^{H} J_{1^{+}}^{\beta_{i}} e_{i}\left(t, u_{t}\right), t \in J, \\
\phi(t), \quad t \in(-\infty, 1] .
\end{array}\right.
$$

Then solving the problem (1) is equivalent to finding the fixed point of the operator $F$ defined by (4). Next we shall present and prove our main results. To this end, the following assumptions are needed later.

$\left(H_{1}\right)$ The functions $e_{i}: J \times \mathcal{B}_{h} \rightarrow \mathbb{R}(i=1,2, \ldots, m)$ are continuous, and there exist some constants $k_{i}>0$ such that

$$
\left|e_{i}(t, u)-e_{i}(t, \bar{u})\right| \leq k_{i}\|u-\bar{u}\|_{\mathcal{B}_{h}}, t \in J, u, \bar{u} \in \mathcal{B}_{h} .
$$

$\left(H_{2}\right)$ The function $a(t) \in C(J, \mathbb{R})$ is not identically zero on any subinterval of $[1, T]$, and there exists a constant $M>0$ such that for any $t \in J,|a(t)| \leq M$.

$\left(H_{3}\right)$ The function $f: J \times \mathcal{B}_{h} \times \mathbb{R} \rightarrow \mathbb{R}$ is continuous, and there exist some constants $n_{1}, n_{2}>0$ such that

$$
|f(t, u, x)-f(t, \bar{u}, y)| \leq n_{1}\|u-\bar{u}\|_{\mathcal{B}_{h}}+n_{2}|x-y|, t \in J, u, \bar{u} \in \mathcal{B}_{h}, x, y \in \mathbb{R} .
$$

$\left(H_{4}\right)$ The function $g: J \times J \times \mathcal{B}_{h} \rightarrow \mathbb{R}$ is continuous, and there exists a constant $q>0$ such that

$$
|g(t, s, u)-g(t, s, \bar{u})| \leq q\|u-\bar{u}\|_{\mathcal{B}_{h}}, t, s \in J, u, \bar{u} \in \mathcal{B}_{h} .
$$

$\left(H_{5}\right) 0<\varrho_{1}<1$, where

$$
\varrho_{1}=l\left[\frac{M(\ln T)^{\alpha}}{\Gamma(\alpha+1)}\left(n_{1}+n_{2} q T\right)+\sum_{i=1}^{m} \frac{k_{i}(\ln T)^{\beta_{i}}}{\Gamma\left(\beta_{i}+1\right)}\right], \quad l=\int_{-\infty}^{0} h(s) d s .
$$

Theorem 1. Assume that $\left(H_{1}\right)-\left(H_{5}\right)$ hold. Then the problem (1) has a unique solution $u^{*} \in \mathbf{X}$. 
Proof. Now we are applying Lemma 3 to prove that $F: \mathbf{X} \rightarrow \mathbf{X}$ defined by (4) has a unique fixed point. Actually, for all $t \in(-\infty, T], u, \bar{u} \in \mathbf{X}$, when $t \in(-\infty, 1]$, we have

$$
(F u)(t)-(F \bar{u})(t)=\phi(t)-\phi(t)=0
$$

which implies that

$$
\|(F u)(t)-(F \bar{u})(t)\|=0 .
$$

When $t \in[1, T]$, it follows from $(4)$ and $\left(H_{1}\right)-\left(H_{5}\right)$ that

$$
\begin{aligned}
& \|(F u)(t)-(F \bar{u})(t)\| \\
& =\| \frac{1}{\Gamma(\alpha)} \int_{1}^{t}\left(\ln \frac{t}{s}\right)^{\alpha-1} a(s)\left[f\left(s, u_{s}, \int_{1}^{s} g\left(s, \tau, u_{\tau}\right) d \tau\right)\right. \\
& \left.-f\left(s, \bar{u}_{s}, \int_{1}^{s} g\left(s, \tau, \bar{u}_{\tau}\right) d \tau\right)\right] \frac{d s}{s}+\sum_{i=1}^{m}{ }^{H} J_{1^{+}}^{\beta_{i}}\left[e_{i}\left(t, u_{t}\right)-e_{i}\left(t, \bar{u}_{t}\right)\right] \| \\
& \leq \frac{1}{\Gamma(\alpha)} \int_{1}^{t}\left(\ln \frac{t}{s}\right)^{\alpha-1}|a(s)|\left(n_{1}\left\|u_{s}-\bar{u}_{s}\right\|_{\mathcal{B}_{h}}+n_{2} \mid \int_{1}^{s} g\left(s, \tau, u_{\tau}\right) d \tau\right. \\
& \left.-\int_{1}^{s} g\left(s, \tau, \bar{u}_{\tau}\right) d \tau \mid\right) \frac{d s}{s}+\sum_{i=1}^{m} \frac{1}{\Gamma\left(\beta_{i}\right)} \int_{1}^{t}\left(\ln \frac{t}{s}\right)^{\beta_{i}-1}\left|e_{i}\left(s, u_{s}\right)-e_{i}\left(s, \bar{u}_{s}\right)\right| \frac{d s}{s} \\
& \leq \frac{1}{\Gamma(\alpha)} \int_{1}^{t}\left(\ln \frac{t}{s}\right)^{\alpha-1}|a(s)|\left(n_{1}\left\|u_{s}-\bar{u}_{s}\right\|_{\mathcal{B}_{h}}+n_{2} \mid \int_{1}^{s}\left[g\left(s, \tau, u_{\tau}\right)\right.\right. \\
& \left.\left.-g\left(s, \tau, \bar{u}_{\tau}\right)\right] d \tau \mid\right) \frac{d s}{s}+\sum_{i=1}^{m} \frac{1}{\Gamma\left(\beta_{i}\right)} \int_{1}^{t}\left(\ln \frac{t}{s}\right)^{\beta_{i}-1} k_{i}\left\|u_{s}-\bar{u}_{s}\right\|_{\mathcal{B}_{h}} \frac{d s}{s} \\
& \leq \frac{M}{\Gamma(\alpha)} \int_{1}^{t}\left(\ln \frac{t}{s}\right)^{\alpha-1}\left(n_{1}\left\|u_{s}-\bar{u}_{s}\right\|_{\mathcal{B}_{h}}+n_{2} \int_{1}^{s} q\left\|u_{\tau}-\bar{u}_{\tau}\right\|_{\mathcal{B}_{h}} d \tau\right) \frac{d s}{s} \\
& +\sum_{i=1}^{m} \frac{k_{i}}{\Gamma\left(\beta_{i}\right)} \int_{1}^{t}\left(\ln \frac{t}{s}\right)^{\beta_{i}-1}\left\|u_{s}-\bar{u}_{s}\right\|_{\mathcal{B}_{h}} \frac{d s}{s} \\
& \leq \frac{M}{\Gamma(\alpha)} \int_{1}^{t}\left(\ln \frac{t}{s}\right)^{\alpha-1}\left(n_{1} l\|u-\bar{u}\|_{T}+n_{2} \int_{1}^{s} q l\|u-\bar{u}\|_{T} d \tau\right) \frac{d s}{s} \\
& +\sum_{i=1}^{m} \frac{k_{i}}{\Gamma\left(\beta_{i}\right)} \int_{1}^{t}\left(\ln \frac{t}{s}\right)^{\beta_{i}-1} l\|u-\bar{u}\|_{T} \frac{d s}{s} \\
& \leq \frac{M}{\Gamma(\alpha)}\left(n_{1} l\|u-\bar{u}\|_{T}+n_{2} q l T\|u-\bar{u}\|_{T}\right) \int_{1}^{t}\left(\ln \frac{t}{s}\right)^{\alpha-1} \frac{d s}{s} \\
& +\sum_{i=1}^{m} \frac{k_{i}}{\Gamma\left(\beta_{i}\right)} l\|u-\bar{u}\|_{T} \int_{1}^{t}\left(\ln \frac{t}{s}\right)^{\beta_{i}-1} \frac{d s}{s} \\
& \leq l\left[\frac{M(\ln T)^{\alpha}}{\Gamma(\alpha+1)}\left(n_{1}+n_{2} q T\right)+\sum_{i=1}^{m} \frac{k_{i}(\ln T)^{\beta_{i}}}{\Gamma\left(\beta_{i}+1\right)}\right]\|u-\bar{u}\| \\
& =\quad \varrho_{1}\|u-\bar{u}\|<\|u-\bar{u}\| .
\end{aligned}
$$

In view of $\left(H_{5}\right),(6)$ and (7), we conclude that $F$ defined as (4) is a contraction mapping. Thus, $F$ has a unique fixed point $u^{*}(t) \in \mathbf{X}$. Consequently, the problem (1) has a unique solution $u^{*}(t) \in \mathbf{X}$. The proof of Theorem 1 is completed.

Theorem 2. Assume that $\left(H_{1}\right)-\left(H_{2}\right)$ hold. If the following conditions $\left(H_{6}\right)-\left(H_{9}\right)$ also hold, then the problem (1) has at least a solution $u^{*}(t) \in \mathbf{X}$.

$\left(H_{6}\right)$ The functions $e_{i}: J \times \mathcal{B}_{h} \rightarrow \mathbb{R}(i=1,2, \ldots, m)$ are continuous functions, and there exist some positive constants $K_{i}$ such that

$$
\left|e_{i}\left(t, u_{t}\right)\right| \leq K_{i}\left\|u_{t}\right\|_{\mathcal{B}_{h}}, t \in J, u_{t} \in \mathcal{B}_{h} .
$$


$\left(H_{7}\right)$ The function $f: J \times \mathcal{B}_{h} \times \mathbb{R} \rightarrow \mathbb{R}$ is a continuous function, and there exist two positive constants $N_{1}, N_{2}$ such that

$$
\left|f\left(t, u_{t}, x\right)\right| \leq N_{1}\left\|u_{t}\right\|_{\mathcal{B}_{h}}+N_{2}|x|, t \in J, u_{t} \in \mathcal{B}_{h}, x \in \mathbb{R} .
$$

$\left(H_{8}\right)$ The function $g: J^{2} \times \mathcal{B}_{h} \rightarrow \mathbb{R}$ is a continuous function, and there exists a positive constant $Q$ such that

$$
\left|g\left(t, s, u_{t}\right)\right| \leq Q\left\|u_{t}\right\|_{\mathcal{B}_{h^{\prime}}} t, s \in J, u_{t} \in \mathcal{B}_{h} .
$$

$\left(H_{9}\right) 0<\varrho_{2}<1$, where

$$
\varrho_{2}=l\left[\frac{M(\ln T)^{\alpha}}{\Gamma(\alpha+1)}\left(N_{1}+N_{2} Q T\right)+\sum_{i=1}^{m} \frac{K_{i}(\ln T)^{\beta_{i}}}{\Gamma\left(\beta_{i}+1\right)}\right], \quad l=\int_{-\infty}^{0} h(s) d s .
$$

Proof. Given a constant $r>0$, define $\bar{\Omega}=\{u \in \mathbf{X}:\|u\| \leq r\}$, where $\|u\|=\|u\|_{\mathcal{B}_{h}^{\prime}}$. It is clear that $\bar{\Omega}$ is a nonempty closed convex subset of the Banach space $\mathbf{X}$. We also define the operator $F: \mathbf{X} \rightarrow \mathbf{X}$ as (4). Here we split $F=F_{1}+F_{2}$ such that, for any $t \in(-\infty, T], u \in \mathbf{X}$,

$$
\begin{gathered}
\left(F_{1} u\right)(t)=\left\{\begin{array}{l}
\frac{1}{\Gamma(\alpha)} \int_{1}^{t}\left(\ln \frac{t}{s}\right)^{\alpha-1} a(s) f\left(s, u_{s}, \int_{1}^{s} g\left(s, \tau, u_{\tau}\right) d \tau\right) \frac{d s}{s}, t \in J, \\
0, t \in(-\infty, 1],
\end{array}\right. \\
\left(F_{2} u\right)(t)=\left\{\begin{array}{l}
\sum_{i=1}^{m} H J_{1}^{\beta_{i}} e_{i}\left(t, u_{t}\right), t \in J, \\
\phi(t), t \in(-\infty, 1] .
\end{array}\right.
\end{gathered}
$$

When $t \in(-\infty, 1]$, for all $u \in \bar{\Omega}$, we have

$$
\|(F u)(t)\|=\|\phi(t)\|=\max \left\{\|\phi(t)\|_{\infty}\right\} \leq\|u\| \leq r,
$$

When $t \in[1, T]$, for all $u \in \bar{\Omega}$, it follows from (4), $\left(H_{2}\right)$ and $\left(H_{6}\right)-\left(H_{9}\right)$ that 


$$
\begin{aligned}
c c\|(F u)(t)\|= & \left\|\frac{1}{\Gamma(\alpha)} \int_{1}^{t}\left(\ln \frac{t}{s}\right)^{\alpha-1} a(s) f\left(s, u_{s}, \int_{1}^{s} g\left(s, \tau, u_{\tau}\right) d \tau\right) \frac{d s}{s}+\sum_{i=1}^{m}{ }^{\beta_{1}} J_{1_{i}} e_{i}\left(t, u_{t}\right)\right\| \\
\leq & \frac{M}{\Gamma(\alpha)} \int_{1}^{t}\left(\ln \frac{t}{s}\right)^{\alpha-1}\left(N_{1}\left\|u_{s}\right\|_{\mathcal{B}_{h}}+N_{2}\left|\int_{1}^{s} g\left(s, \tau, u_{\tau}\right) d \tau\right|\right) \frac{d s}{s} \\
& +\sum_{i=1}^{m} \frac{1}{\Gamma\left(\beta_{i}\right)} \int_{1}^{t}\left(\ln \frac{t}{s}\right)^{\beta_{i}-1}\left|e_{i}\left(s, u_{s}\right)\right| \frac{d s}{s} \\
\leq & \frac{M}{\Gamma(\alpha)} \int_{1}^{t}\left(\ln \frac{t}{s}\right)^{\alpha-1}\left(N_{1}\left\|u_{s}\right\|_{\mathcal{B}_{h}}+N_{2} Q \int_{1}^{s}\left\|u_{\tau}\right\|_{\mathcal{B}_{h}}\right) d \tau \frac{d s}{s} \\
& +\sum_{i=1}^{m} \frac{1}{\Gamma\left(\beta_{i}\right)} \int_{1}^{t}\left(\ln \frac{t}{s}\right)^{\beta_{i}-1} K_{i}\left\|u_{s}\right\|_{\mathcal{B}_{h}} \frac{d s}{s} \\
\leq & \frac{M}{\Gamma(\alpha)}\left(N_{1} l\|u\|_{T}+N_{2} Q T l\|u\|_{T}\right) \int_{1}^{t}\left(\ln \frac{t}{s}\right)^{\alpha-1} \frac{d s}{s} \\
& +\sum_{i=1}^{m} \frac{1}{\Gamma\left(\beta_{i}\right)} K_{i} l\|u\|_{T} \int_{1}^{t}\left(\ln \frac{t}{s}\right)^{\beta_{i}-1} \frac{d s}{s} \\
\leq & {\left[\frac{M(\ln T)^{\alpha}}{\Gamma(\alpha+1)}\left(N_{1}+N_{2} Q T\right)+\sum_{i=1}^{m} \frac{K_{i}(\ln T)^{\beta_{i}}}{\Gamma\left(\beta_{i}+1\right)}\right]\|u\|_{T} } \\
\leq & \varrho_{2}\|u\|<\|u\| \leq r .
\end{aligned}
$$

We derive that $F u=F_{1} u+F_{2} u \in \bar{\Omega}$ from (10) and (11), that is, the condition (i) of Lemma 4 holds. Similarly, we can also prove that $F_{1}, F_{2}: \bar{\Omega} \rightarrow \bar{\Omega} \subset \mathbf{X}$, and $F_{1}$ is uniformly bounded.

Next we only need to prove that $F_{1}$ is equicontinuous, and $F_{2}$ is a contraction mapping. In fact, for all $u, \bar{u} \in \bar{\Omega}$, when $t \in(-\infty, 1]$,

$$
\left(F_{2} u\right)(t)-\left(F_{2} \bar{u}\right)(t)=\phi(t)-\phi(t)=0,
$$

which implies that

$$
\left\|\left(F_{2} u\right)(t)-\left(F_{2} \bar{u}\right)(t)\right\|=0 .
$$

When $t \in[1, T]$, it follows from (9), $\left(H_{1}\right)$ and $\left(H_{5}\right)$ that

$$
\begin{aligned}
& c c\left\|\left(F_{2} u\right)(t)-\left(F_{2} \bar{u}\right)(t)\right\|=\left\|\sum_{i=1}^{m}{ }^{H} J_{1^{+}}^{\beta_{i}}\left[e_{i}\left(t, u_{t}\right)-e_{i}\left(t, \bar{u}_{t}\right)\right]\right\| \\
& \leq \sum_{i=1}^{m} \frac{1}{\Gamma\left(\beta_{i}\right)} \int_{1}^{t}\left(\ln \frac{t}{s}\right)^{\beta_{i}-1}\left|e_{i}\left(s, u_{s}\right)-e_{i}\left(s, \bar{u}_{s}\right)\right| \frac{d s}{s} \\
& \leq \sum_{i=1}^{m} \frac{1}{\Gamma\left(\beta_{i}\right)} \int_{1}^{t}\left(\ln \frac{t}{s}\right)^{\beta_{i}-1} k_{i}\left\|u_{s}-\bar{u}_{s}\right\|_{\mathcal{B}_{h}} \frac{d s}{s} \\
& \leq \sum_{i=1}^{m} \frac{k_{i}}{\Gamma\left(\beta_{i}\right)} \int_{1}^{t}\left(\ln \frac{t}{s}\right)^{\beta_{i}-1} l\|u-\bar{u}\|_{T} \frac{d s}{s} \\
& \leq \sum_{i=1}^{m} \frac{k_{i}}{\Gamma\left(\beta_{i}\right)} l\|u-\bar{u}\|_{T} \int_{1}^{t}\left(\ln \frac{t}{s}\right)^{\beta_{i}-1} \frac{d s}{s} \\
& \leq \sum_{i=1}^{m} \frac{k_{i} l(\ln T)^{\beta_{i}}}{\Gamma\left(\beta_{i}+1\right)}\|u-\bar{u}\|<\varrho_{1}\|u-\bar{u}\|<\|u-\bar{u}\| .
\end{aligned}
$$


(13) and (14) means that $F_{2}$ is a contraction mapping. Therefore, the condition (ii) of Lemma 4 holds. Finally, we show that $F_{1}$ is equicontinuous. For all $u \in \bar{\Omega}, t_{1}, t_{2} \in(-\infty, T]$ with $t_{1}<t_{2}$, when $-\infty<t_{1}<t_{2} \leq 1$, from (8),

$$
\left\|\left(F_{1} u\right)\left(t_{2}\right)-\left(F_{1} u\right)\left(t_{1}\right)\right\|=0 \rightarrow 0, \text { as } t_{2} \rightarrow t_{1} .
$$

When $1 \leq t_{1}<t_{2} \leq T$, from (8),

$$
\begin{aligned}
& c c\left\|\left(F_{1} u\right)\left(t_{2}\right)-\left(F_{1} u\right)\left(t_{1}\right)\right\| \\
& =\| \frac{1}{\Gamma(\alpha)} \int_{1}^{t_{2}}\left(\ln \frac{t_{2}}{s}\right)^{\alpha-1} a(s) f\left(s, u_{s}, \int_{1}^{s} g\left(s, \tau, u_{\tau}\right) d \tau\right) \frac{d s}{s} \\
& -\frac{1}{\Gamma(\alpha)} \int_{1}^{t_{1}}\left(\ln \frac{t_{1}}{s}\right)^{\alpha-1} a(s) f\left(s, u_{s}, \int_{1}^{s} g\left(s, \tau, u_{\tau}\right) d \tau\right) \frac{d s}{s} \| \\
& =\| \frac{1}{\Gamma(\alpha)} \int_{1}^{t_{1}}\left[\left(\ln \frac{t_{2}}{s}\right)^{\alpha-1}-\left(\ln \frac{t_{1}}{s}\right)^{\alpha-1}\right] a(s) f\left(s, u_{s}, \int_{1}^{s} g\left(s, \tau, u_{\tau}\right) d \tau\right) \frac{d s}{s} \\
& +\frac{1}{\Gamma(\alpha)} \int_{t_{1}}^{t_{2}}\left(\ln \frac{t_{2}}{s}\right)^{\alpha-1} a(s) f\left(s, u_{s}, \int_{1}^{s} g\left(s, \tau, u_{\tau}\right) d \tau\right) \frac{d s}{s} \| \\
& \leq \frac{M}{\Gamma(\alpha)} \int_{1}^{t_{1}}\left[\left(\ln \frac{t_{2}}{s}\right)^{\alpha-1}-\left(\ln \frac{t_{1}}{s}\right)^{\alpha-1}\right]\left(N_{1}\left\|u_{s}\right\|_{\mathcal{B}_{h}}+N_{2}\left|\int_{1}^{s} g\left(s, \tau, u_{\tau}\right) d \tau\right|\right) \frac{d s}{s} \\
& +\frac{M}{\Gamma(\alpha)} \int_{t_{1}}^{t_{2}}\left(\ln \frac{t_{2}}{s}\right)^{\alpha-1}\left(N_{1}\left\|u_{s}\right\|_{\mathcal{B}_{h}}+N_{2}\left|\int_{1}^{s} g\left(s, \tau, u_{\tau}\right) d \tau\right|\right) \frac{d s}{s} \\
& \leq \frac{M}{\Gamma(\alpha)} \int_{1}^{t_{1}}\left[\left(\ln \frac{t_{2}}{s}\right)^{\alpha-1}-\left(\ln \frac{t_{1}}{s}\right)^{\alpha-1}\right]\left(N_{1}\left\|u_{s}\right\|_{\mathcal{B}_{h}}+N_{2} Q \int_{1}^{s}\left\|u_{\tau}\right\|_{\mathcal{B}_{h}} d \tau\right) \frac{d s}{s} \\
& +\frac{M}{\Gamma(\alpha)} \int_{t_{1}}^{t_{2}}\left(\ln \frac{t_{2}}{s}\right)^{\alpha-1}\left(N_{1}\left\|u_{s}\right\|_{\mathcal{B}_{h}}+N_{2} Q \int_{1}^{s}\left\|u_{\tau}\right\|_{\mathcal{B}_{h}} d \tau\right) \frac{d s}{s} \\
& \leq \frac{M}{\Gamma(\alpha)} \int_{1}^{t_{1}}\left[\left(\ln \frac{t_{2}}{s}\right)^{\alpha-1}-\left(\ln \frac{t_{1}}{s}\right)^{\alpha-1}\right]\left(N_{1}\left\|u_{s}\right\|_{\mathcal{B}_{h}}+N_{2} Q T\left\|u_{\tau}\right\|_{\mathcal{B}_{h}}\right) \frac{d s}{s} \\
& +\frac{M}{\Gamma(\alpha)} \int_{t_{1}}^{t_{2}}\left(\ln \frac{t_{2}}{s}\right)^{\alpha-1}\left(N_{1}\left\|u_{s}\right\|_{\mathcal{B}_{h}}+N_{2} Q T\left\|u_{\tau}\right\|_{\mathcal{B}_{h}}\right) \frac{d s}{s} \\
& \leq \frac{M l}{\Gamma(\alpha)}\left(N_{1}+N_{2} Q T\right)\|u\|_{T} \int_{1}^{t_{1}}\left[\left(\ln \frac{t_{2}}{s}\right)^{\alpha-1}-\left(\ln \frac{t_{1}}{s}\right)^{\alpha-1}\right] \frac{d s}{s} \\
& +\frac{M l}{\Gamma(\alpha)}\left(N_{1}+N_{2} Q T\right)\|u\|_{T} \int_{t_{1}}^{t_{2}}\left(\ln \frac{t_{2}}{s}\right)^{\alpha-1} \frac{d s}{s} \\
& \leq \frac{M l}{\Gamma(\alpha)}\left(N_{1}+N_{2} Q T\right)\|u\| \int_{1}^{t_{1}}\left[\left(\ln \frac{t_{2}}{s}\right)^{\alpha-1}-\left(\ln \frac{t_{1}}{s}\right)^{\alpha-1}\right] \frac{d s}{s} \\
& +\frac{M l}{\Gamma(\alpha+1)}\left(N_{1}+N_{2} Q T\right)\|u\|\left(\ln \frac{t_{2}}{t_{1}}\right)^{\alpha} \rightarrow 0 \text {, as } t_{2} \rightarrow t_{1} \text {. }
\end{aligned}
$$

When $-\infty<t_{1}<1<t_{2} \leq T$, then $t_{2} \rightarrow t_{1}$ means that $t_{1} \rightarrow 1^{-}$and $t_{2} \rightarrow 1^{+}$. We can obtain that

$$
\left\|\left(F_{1} u\right)\left(t_{2}\right)-\left(F_{1} u\right)\left(t_{1}\right)\right\| \rightarrow\|0-0\|=0 \text {, as } t_{2} \rightarrow t_{1} .
$$

(15)-(17) implies that $F_{1}$ is equicontinuous. By the Arzelá-Ascoli theorem, we conclude that $F_{1}: \bar{\Omega} \rightarrow \bar{\Omega}$ is completely continuous, that is, the condition (iii) of Lemma 4 holds. According to Lemma 4, we derive that the problem (1) has at least a solution $u^{*}(t) \in \mathbf{X}$. The proof is completed. 


\section{Illustrative Example}

Consider the following nonlinear neutral Hadamard fractional integro-differential equation with infinite delay of the form

$$
\left\{\begin{array}{l}
{ }^{H} D_{1^{+}}^{\frac{1}{2}}\left[u(t)-\sum_{i=1}^{2} H J_{1^{+}}^{\frac{2 i+1}{2}} e_{i}\left(t, u_{t}\right)\right]=a(t) f\left(t, u_{t}, \int_{1}^{t} g\left(t, s, u_{s}\right) d s\right), t \in J=[1, e], \\
u(t)=\phi(t), t \in(-\infty, 1],
\end{array}\right.
$$

where $\alpha=\frac{1}{2}, \beta_{i}=\frac{2 i+1}{2}, i=1,2, m=2, u_{t}(\theta)=u(t+\theta), \theta \leq 0,{ }^{H} D_{1^{+}}^{\alpha}$ is the Hadamard fractional derivative of order $\alpha$ and ${ }^{H} J_{1^{+}}^{\beta_{i}}$ is the Hadamard fractional integral of order $\beta_{i}$.

Let $h(s)=e^{2 s}, s<0$, then $l=\int_{-\infty}^{0} h(s) d s=\frac{1}{2}<\infty$. Thus, we easily obtain $\mathcal{B}, \mathcal{B}_{h}$, $\mathcal{B}_{h}^{\prime}=\mathbf{X}$ and their norms from the definitions of $\mathcal{B}, \mathcal{B}_{h}, \mathcal{B}_{h}^{\prime}$. Take $e_{i}\left(t, u_{t}\right)=\frac{u_{t} \sin t}{50 i}(i=1,2)$, $a(t)=\frac{t(3-t)}{10}, f\left(t, u_{t}, x\right)=\frac{u_{t}^{2} \cos 2 t+x}{180}, g\left(t, s, u_{s}\right)=\frac{e^{-(t+s)} u_{s}}{150}$ and $\phi(t)=\sin t$. By a simple calculation, for $t \in J, u_{t}, \bar{u}_{t} \in \mathcal{B}_{h}, x, y \in \mathbb{R}$, we have

$$
\begin{gathered}
\left|e_{i}\left(t, u_{t}\right)-e_{i}\left(t, \bar{u}_{t}\right)\right| \leq \frac{1}{50 i}\left\|u_{t}-\bar{u}_{t}\right\|_{\mathcal{B}_{h^{\prime}}} i=1,2, \\
\left|f\left(t, u_{t}, x\right)-f\left(t, \bar{u}_{t}, y\right)\right| \leq \frac{1}{90}\left\|u_{t}-\bar{u}_{t}\right\|_{\mathcal{B}_{h}}+\frac{1}{180}|x-y|, \\
\left|g\left(t, s, u_{t}\right)-g\left(t, s, \bar{u}_{t}\right)\right| \leq \frac{1}{150}\left\|u_{t}-\bar{u}_{t}\right\|_{\mathcal{B}_{h} .}
\end{gathered}
$$

We can easily obtain $|a(t)| \leq \frac{9}{40}$. Hence, from the above calculations, we know $l=\frac{1}{2}$, $T=e, k_{1}=\frac{1}{50}, k_{2}=\frac{1}{100}, M=\frac{9}{40}, n_{1}=\frac{1}{90}, n_{2}=\frac{1}{180}, q=\frac{1}{150}$. Noticing that $\Gamma\left(\frac{1}{2}\right)=\sqrt{\pi}$, then we derive

$$
0<\varrho_{1}=l\left[\frac{M(\ln T)^{\alpha}}{\Gamma(\alpha+1)}\left(n_{1}+n_{2} q T\right)+\sum_{i=1}^{m} \frac{k_{i}(\ln T)^{\beta_{i}}}{\Gamma\left(\beta_{i}+1\right)}\right] \approx 0.0165<1 .
$$

Thus the assumptions $\left(H_{1}\right)-\left(H_{5}\right)$ of Theorem (1) hold. According to Theorem (1), We know that the problem (18) has a unique solution $u^{*} \in \mathbf{X}$.

\section{Conclusions}

As a useful mathematical model, fractional-order differential calculus is used to describe properties of various processes and applications in lots of fields such as blood flow problems, chemical engineering, porous medium, aerodynamics, polymer rheology, population dynamics, and so on. Compared with Caputo or Riemann-Liouville type fractional differential equations, the study on Hadamard-type fractional differential equations is more difficult and complicated. The study on Hadamard-type fractional differential equations involving the time delays will be more challenging. Therefore, we mainly study the existence of solutions of the problem (1) in this paper. By applying the Banach contraction principle and Krasnoselskii's fixed point theorem, some new existence criteria of solutions are obtained.

Author Contributions: Conceptualization, K.Z. and Y.M.; Methodology, K.Z. and Y.M.; Validation, Y.M.; Formal analysis, K.Z. and Y.M.; Investigation, K.Z. and Y.M.; Resources, Y.M.; Writing-original draft preparation, Y.M.; Writing—review and editing, K.Z.; Visualization, Y.M.; Supervision, K.Z.; Project administration, Y.M.; Funding acquisition, Y.M. All authors have read and agreed to the published version of the manuscript.

Funding: This work was supported by the National Natural Sciences Foundation of Peoples Republic of China under Grant (No. 11661047). 
Institutional Review Board Statement: Not applicable.

Informed Consent Statement: Not applicable.

Data Availability Statement: Not applicable.

Acknowledgments: The authors would like to thank the anonymous referees for their useful and valuable suggestions.

Conflicts of Interest: The authors declare that they have no competing interests.

\section{References}

1. Dabas, J.; Chauhan, A. Existence and uniqueness of mild solution for an impulsive neutral fractional integro-differential equation with infinite delay. Math. Comput. Model. 2013, 57, 754-763. [CrossRef]

2. Chang, Y.; Anguraj, A.; Arjunan, M. Existence results for impulsive neutral functional differential equations with infinite delay. Nonlinear Anal. Hybrid Syst. 2008, 2, 209-218. [CrossRef]

3. Cao, J.; Luo, Y.; Liu, G. Some results for impulsive fractional differential inclusions with infinite delay and sectorial operators in Banach spaces. Appl. Math. Comput. 2016, 273, 237-257. [CrossRef]

4. Bao, H.; Cao, J. Existence of solutions for fractional stochastic impulsive neutral functional differential equations with infinite delay. Adv. Differ. Equ. 2017, 1, 66. [CrossRef]

5. Zhao, K.; Suo, L.; Liao, Y. Boundary value problem for a class of fractional integro-differential coupled systems with hadamard fractional calculus and impulses. Bound. Value Probl. 2019, 1, 105. [CrossRef]

6. Kalamani, P.; Baleanu, D.; Arjunan, M. Local existence for an impulsive fractional neutral integro-differential system with Riemann-Liouville fractional derivatives in a Banach space. Adv. Differ. Equ. 2018, 1, 416. [CrossRef]

7. Zhao, K. Triple positive solutions for two classes of delayed nonlinear fractional FDEs with nonlinear integral boundary value conditions. Bound. Value Probl. 2015, 1, 181. [CrossRef]

8. Zhao, K. Multiple positive solutions of integral BVPs for high-order nonlinear fractional differential equations with impulses and distributed delays. Dyn. Syst. 2015, 30, 208-223. [CrossRef]

9. Shah, K.; Khan, R.; Baleanu, D. Study of implicit type coupled system of non-integer order differential equations with anti-periodic boundary conditions. Math. Methods Appl. Sci. 2019. [CrossRef]

10. Shiri, B.; Baleanu, D. System of fractional differential algebraic equations with applications. Chaos Solitons Fractals 2019, 120, 203-212. [CrossRef]

11. Zhou, Y.; Jiao, F. Existence of mild solutions for fractional neutral evolution equations Comput. Math. Appl. 2010, 59, 1063-1077.

12. Cichon, M.; Salem, H.A.H. On the lack of equivalence between differential and integral forms of the Caputo-type fractional problems. J. Pseudo-Differ. Oper. Appl. 2020, 11, 1869-1895. [CrossRef]

13. Hadamard, J. Essai sur L'étude des fonctions données par leur développment de Taylor. Math. Pures Appl. 1892, 8, 101-186.

14. Kilbas, A.; Srivastava, H.; Trujillo, J. Theory and Applications of Fractional Differential Equations; North-Holland Mathematics Studies; Elsevier: Amsterdam, The Netherlands, 2006; Volume 204.

15. Ahmad, B.; Alsaedi, A.; Ntouyas, S.; Tariboon, J. Hadamard-Type Fractional Differential Equations, Inclusions and Inequalities; Springer: Berlin/Heidelberg, Germany, 2017.

16. Butzer, P.; Kilbas, A.; Trujillo, J. Compositions of Hadamard-type fractional integration operators and the semigroup property. Math. Appl. Anal. 2002, 269, 387-400. [CrossRef]

17. Butzer, P.; Kilbas, A.; Trujillo, J. Fractional calculus in the Mellin setting and Hadamard-type fractional integrals. Math. Appl. Anal. 2002, 269, 1-27. [CrossRef]

18. Ahmad, B.; Ntouyas, S. On Hadamard fractional integro-differential boundary value problems. Appl. Math. Comput. 2015, 47, 119-131. [CrossRef]

19. Ahmad, B.; Ntouyas, S. Boundary value problems of Hadamard-type fractional differential equations and inclusions with nonlocal conditions. Vietnam J. Math. 2017, 45, 409-423. [CrossRef]

20. Salem, A.H. Hadamard-type fractional calculus in Banach spaces. RACSAM Rev. R. Acad. A 2019, 113, 987-1006. [CrossRef]

21. Abdalla, A.M.; Salem, H.A.H.; Cichon, K. On positive solutions of a system of equations generated by Hadamard fractional operators. Adv. Differ. Equ. 2020, 1, 267. [CrossRef]

22. Cichon, M.; Salem, H.A.H. On the solutions of Caputo-Hadamard Pettis-type fractional differential equations. RACSAM Rev. R. Acad. A 2019, 113, 3031-3053. [CrossRef]

23. Yukunthorn, W.; Suantai, S.; Ntouyas, S.; Tariboon, J. Boundary value problems for impulsive multi-order Hadamard fractional differential equations. Bound. Value Probl. 2015, 1, 148. [CrossRef]

24. Abbas, M.I. On the Hadamard and Riemann-Liouville fractional neutral functional integrodifferential equations with finite delay. J. Pseudo-Differ. Oper. Appl. 2018. [CrossRef]

25. Aljoudi, S.; Ahmad, B.; Nieto, J.; Alsaedi, A. A coupled system of Hadamard type sequential fractional differential equations with coupled strip conditions. Chaos Soliton Fract. 2016, 91, 39-46. [CrossRef]

26. Chang, Y. Controllability of impulsive functional differential systems with infinite delay in Banach spaces. Chaos Soliton Fract. 2007, 33, 1601-1609. [CrossRef] 
27. Guo, D.; Lakshmikantham, V. Nonlinear Problems in Abstract Cone; Academic Press: Orlando, FL, USA, 1988.

28. Granas, A.; Dugundji, J. Fixed Point Theory; Springer: New York, NY, USA, 2003. 